\title{
Psicooncología
}

ISSN: 1696-7240

https://dx.doi.org/10.5209/psic.74535

\section{Desarrollo de una estrategia holística para la valoración de la calidad de vida en pacientes con cáncer de mama en las distintas etapas de la enfermedad}

Lorena Gómez-Villarroya ${ }^{1}$; Clara Serra-Arumí2; Coral Báez-Sáez ${ }^{3}$; Marisa Mena Cervigon ${ }^{4}$; Sara Tous Belmonte ${ }^{5}$; Francisca Morey Cortes $^{6}$; Eva Rodríguez Bruzos ${ }^{7}$; Miguel Gil Gil ${ }^{8}$; Sonia Pernas Simón"; Andrea Vethencourt Casado ${ }^{10}$; Silvia Vázquez Fernández ${ }^{11}$; Agostina Stradella ${ }^{12}$; Catalina Falo Zamora ${ }^{13}$; Antoni Font Guiteras ${ }^{14 *}$

1 Lorena Gómez-Villarroya. Facultad de Psicología, Universitat Autònoma de Barcelona. Barcelona.

E-mail: lorena.gomezv@e-campus.uab.cat

2 Clara Serra-Arumí. Facultad de Psicología, Universitat Autònoma de Barcelona. Barcelona. Teaching, Research and Innovation Unit. Institut de Recerca Sant Joan de Déu. Esplugues de Llobregat. Barcelona.

E-mail: clara.serraa@e-campus.uab.cat

3 Coral Báez-Sáez. Facultad de Psicología, Universitat Autònoma de Barcelona. Barcelona.

E-mail: coral.baez@e-campus.uab.cat

4 Marisa Mena Cervigon. Cancer Epidemiology Research Program (CERP), Institut Català d'Oncologia. L'Hospitalet de Llobregat, Barcelona. Epidemiology, Public Health, Cancer Prevention and Palliative Care Program. Institut d'Investigació Biomèdica de Bellvitge (IDIBELL). L'Hospitalet de Llobregat. Barcelona.

E-mail: mmena.iconcologia@gmail.com

5 Sara Tous Belmonte. Cancer Epidemiology Research Program (CERP), Institut Català d'Oncologia. L'Hospitalet de Llobregat, Barcelona. Epidemiology, Public Health, Cancer Prevention and Palliative Care Program. Institut d’Investigació Biomèdica de Bellvitge (IDIBELL). L'Hospitalet de Llobregat. Barcelona.

E-mail: stous@iconcologia.net

6 Francisca Morey Cortes. Cancer Epidemiology Research Program (CERP), Institut Català d'Oncologia. L'Hospitalet de Llobregat, Barcelona. Epidemiology, Public Health, Cancer Prevention and Palliative Care Program. Institut d'Investigació Biomèdica de Bellvitge (IDIBELL). L'Hospitalet de Llobregat. Barcelona.

E-mail: fmorey@idibell.cat

7 Eva Rodríguez Bruzos. Facultad de Psicología, Universitat Autònoma de Barcelona. Barcelona.

E-mail: evarb71@hotmail.com

8 Miguel Gil Gil. Servicio de Oncología Médica. Institut Català d'Oncologia (IDIBELL). L'Hospitalet de Llobregat, Barcelona.

E-mail: mgilgil@iconcologia.net

9 Sonia Pernas Simón. Servicio de Oncología Médica. Institut Català d’Oncologia (IDIBELL). L’Hospitalet de Llobregat, Barcelona.

E-mail: spernas@iconcologia.net

10 Andrea Vethencourt Casado. Servicio de Oncología Médica. Institut Català d'Oncologia (IDIBELL). L'Hospitalet de Llobregat, Barcelona.

E-mail: acvethencourt@iconcologia.net

11 Silvia Vázquez Fernández. Servicio de Oncología Médica. Institut Català d'Oncologia (IDIBELL). L’Hospitalet de Llobregat, Barcelona.

E-mail: silviavazquez@iconcologia.net

12 Agostina Stradella. Servicio de Oncología Médica. Institut Català d'Oncologia (IDIBELL). L’Hospitalet de Llobregat, Barcelona.

E-mail: astradella@iconcologia.net

13 Catalina Falo Zamora. Servicio de Oncología Médica. Institut Català d'Oncologia (IDIBELL). L'Hospitalet de Llobregat, Barcelona.

E-mail: cfalo@iconcologia.net

14 Antoni Font Guiteras. Facultad de Psicología, Universitat Autònoma de Barcelona. Barcelona. z

E-mail: Antonio.Font@uab.cat

* Dirección de correspondencia: Antoni Font Guiteras. Universitat Autònoma de Barcelona. Facultat de Psicologia. Edifici B. Despatx B5b-073 Campus de la UAB · 08193 Bellaterra (Cerdanyola del Vallès) $\cdot$ Barcelona Spain. E-mail: Antonio.Font@uab.cat 
Resumen: Introducción: El cáncer de mama es considerado una enfermedad crónica que impacta de modo importante en la calidad de vida (QoL) de las pacientes. En la actualidad no se dispone de un sistema de evaluación totalmente satisfactorio que refleje la complejidad del cáncer de mama. Además, la mayoría de instrumentos están más orientados a la enfermedad en estadios iniciales que a la enfermedad avanzada. Objetivo: Describir la metodología utilizada para evaluar la calidad de vida y las estrategias de afrontamiento en un grupo representativo de cáncer de mama que incluye tanto pacientes metastásicas como no metastásicas. Método: Estudio prospectivo en pacientes con cáncer de mama del Servicio de Oncología Médica del Institut Català d'Oncologia (ICO) en diferentes estadios de la enfermedad, previo consentimiento informado. Se les aplica un protocolo de aproximadamente una hora de entrevista presencial donde se recoge su información sociodemográfica, y se contestan varios cuestionarios de calidad de vida como el cuestionario QLCA-AFex Font, QLQ-C30, QLQ-BR23 HADS, DME, BRCS, MINI-MAC, LOT-R y OE, que se completan con una entrevista semiestructurada. Resultados: De junio de 2017 a marzo de 2020, 257 pacientes han sido incluidas en el estudio. La media de edad es de 57,9 años (SD 10,1), la mayoría son mujeres $(98,8 \%)$, con hijos $(87,9 \%)$ y casadas $(65,4 \%)$. Respecto al estadiaje clínico $75,5 \%$ son no-metastásicas y $24,5 \%$ metastásicas. El cumplimiento del protocolo se consiguió en más del $90 \%$ de los cuestionarios sin diferencias entre pacientes metastásicas y no metastásicas. Conclusión: Este protocolo multidimensional permite hacer una evaluación integral de la calidad de vida, así como reflejar las necesidades no atendidas y las preocupaciones que muestran las pacientes tanto en estadio precoz como avanzado, complementando los sistemas de valoración actualmente disponibles. Después del análisis de los resultados de este estudio sería interesante poder obtener un cuestionario único con las preguntas más relevantes que pudiera ser aplicado a la práctica clínica.

Palabras clave: Calidad de vida, cáncer de mama, enfermedad avanzada, protocolo, enfoque multidimensional, necesidades no atendidas.

\title{
[en] Development of a holistic strategy for the assessment of the quality of life in patients with breast cancer in the different stages of the disease
}

\begin{abstract}
Introduction: Breast cancer is considered a chronic disease that has considerable impact on the quality of life (QoL) of the patients. Yet, there is not a totally satisfactory evaluation system reflecting the complexity of the breast cancer condition. Furthermore, most instruments are more addressed towards early-stage disease than advanced disease. Objective: To describe the methodology used to measure the QoL of life and coping strategies in a representative group of breast cancer patients including metastatic and non-metastatic patients. Methodology: Prospective study including patients with breast cancer at different stages of the disease recruited at the Medical Oncology Department of the Catalan Oncology Institute (ICO) previous informed consent. A protocol of approximately one hour face-to-face interview is run to collect sociodemographic information and to answer the questions of several QoL scales such as the QLCA-AFex Font, QLQ-C30, QLQ-BR23, HADS, DME, $B R C S$, MINI-MAC, LOT-R and $O E$ questionnaires, complemented by a semi-structured interview. Results: From June 2017 to March 2020, 257 patients were included in the study. Mean age 57.9 years (SD 10.1), mostly women (98.8\%), with children (87.9\%) and married (65.4\%). According to clinical status $75.5 \%$ were non-metastatic and $24.5 \%$ metastatic. Protocol compliance was achieved in more than $90 \%$ in all questionnaires without differences between metastatic and no metastatic patients. Conclusions: This multidimensional protocol enables us to make an integral assessment of the QoL of the patients and their unmet needs, as well as to reflect the patients' concerns, both in early and advanced stages of the disease, complementing currently available assessment methods. In the next future a complied questionnaire with the most challenging questions could be developed to be useful as a routine instrument in clinical practice.
\end{abstract}

Keywords: Quality of life, breast cancer, advanced disease, protocol, multidimensional approach, unmet needs.

Sumario. 1. Introducción 2. Método 3. Resultados 4. Discusión 5. Conclusión y perspectivas de futuro 6. Agradecimientos 7. Financiación 8. Referencias bibliográficas. 
Cómo citar: Gómez-Villarroya L, Serra-Arumí C, Báez-Sáez C, Mena Cervigon M, Tous Belmonte S, Morey Cortes F, Rodríguez Bruzos E, Gil Gil M, Pernas Simón S, Vethencourt Casado A, Vázquez Fernández S, Stradella A, Falo Zamora C, Font Guiteras A, Desarrollo de una estrategia holística para la valoración de la calidad de vida en pacientes con cáncer de mama en las distintas etapas de la enfermedad. Psicooncología 2021; 18: 117-136. doi:10.5209/psic.74535

\section{Introducción}

El cáncer es un problema de salud pública a nivel mundial que no sólo pone en peligro la salud física de quien lo padece, sino que también perturba su salud psicológica y social ${ }^{(1)}$. Entre los diferentes tipos de cáncer, la neoplasia de mama es la más frecuente entre las mujeres ${ }^{(2)}$. Gracias a los avances en detección precoz y en los tratamientos, la tasa de supervivencia global a los cinco años del diagnóstico es del $82,8 \%{ }^{(3)}$, lo que resulta en un número cada vez mayor de supervivientes a largo plazo $^{(4,5)}$. Por otra parte, en las pacientes con enfermedad avanzada, ya sea al debut o tras un intervalo libre de enfermedad, también se ha conseguido prolongar el tiempo de supervivencia y por lo tanto de convivencia con la enfermedad, que puede ser mayor o menor en función del subtipo molecular y la localización de las metástasis, pero que, en cualquier caso, se ha doblado en los últimos 15 años $^{(6)}$. Esta nueva situación exige a los profesionales hacer un esfuerzo en conocer la calidad de vida a lo largo de la enfermedad de las pacientes (tanto en enfermedad precoz como avanzada) y profundizar en cómo les afecta psicológicamente dicha enfermedad, sobre todo en aquellas en fase avanzada, ya que muchas veces se sienten olvidadas por la comunidad médica y científica ${ }^{(7)}$.

Dado que cada vez más mujeres sobreviven al cáncer de mama, la calidad de vida se ha convertido en uno de los focos principales de la investigación y la práctica clínica ${ }^{(8)}$. Además, estas pacientes, independientemente de la etapa en la que se encuentren, consideran la calidad de vida una preocupación crucial $^{(9)}$ que se relaciona de manera importante con su percepción de salud ${ }^{(10)}$.

En la literatura está descrito que las pacientes en estadios precoces presentan síntomas que mayoritariamente disminuyen a medida que pasa el tiempo y mejoran sus circunstancias clínicas ${ }^{(11,12)}$, mientras que las mujeres que se enfrentan a un diagnóstico de cáncer avanzado muestran un mayor número de síntomas físicos $\mathrm{y}$ emocionales, con el agravante de que sus expectativas de futuro empeoran con el tiempo y, con ellas, disminuye progresivamente su calidad de vida ${ }^{(13)}$.

En un estudio previo basado en la calidad de vida en pacientes con cáncer de mama metastásico ${ }^{(13)}$, evidenciamos que la percepción de la calidad de vida no era proporcional a la gravedad de la situación clínica de las pacientes. Para confirmar dicho resultado exploratorio, decidimos diseñar un estudio prospectivo, longitudinal y realizado por un equipo multidisciplinar en el ámbito hospitalario, que incluyera pacientes en diferentes estadios de la enfermedad, la metodología del cual se expone detalladamente en el presente trabajo. 


\section{Método}

Estamos llevando a cabo una investigación prospectiva, de diseño longitudinal y correlacional, con tres recogidas transversales y con una cadencia entre 6 y 12 meses, según la situación clínica de las pacientes. En el presente estudio se aportan datos correspondientes a la primera valoración de las pacientes.

\section{Participantes}

Criterios de inclusión

Pacientes con cáncer de mama del Servicio de Oncología Médica del Institut Català d'Oncologia (ICO) de l'Hospitalet de Llobregat, Barcelona, España, independiente de su estadio de enfermedad. Las pacientes diagnosticadas en estadios iniciales son incluidas en el estudio tras haber finalizado los tratamientos activos de quimioterapia y/o radioterapia. Mientras que las pacientes metastásicas son todas incluidas desde el momento del diagnóstico de enfermedad avanzada. Además, en el presente estudio se han recogido los datos de los pacientes de sexo masculino ya que, a pesar de que hay una menor incidencia en dicho grupo poblacional (menos del $1 \%)^{(14)}$, se considera enriquecedor tenerlos en cuenta para contemplar la realidad que se observa en la práctica clínica*.

Figura 1. Diagrama inclusión-exclusión de participantes

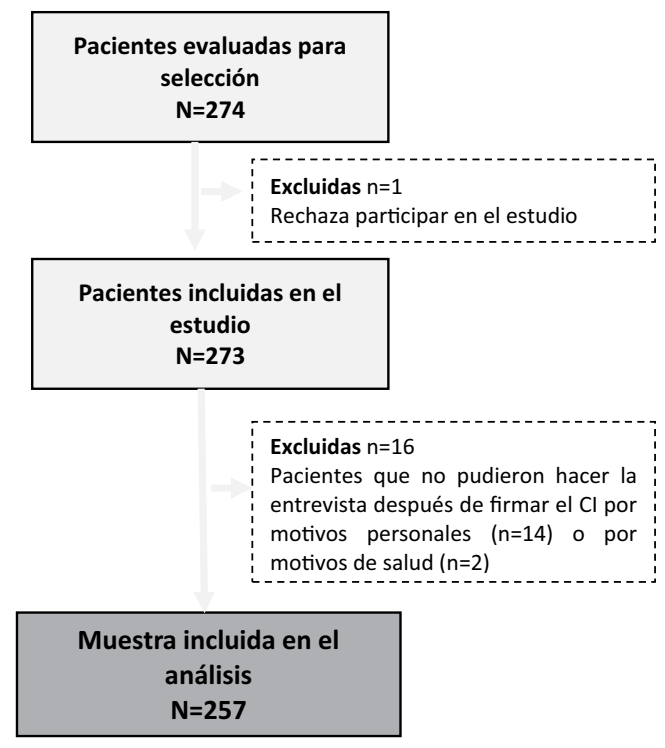

Criterios de exclusión

Pacientes en estadios iniciales que se encontraban en tratamiento activo de quimioterapia y/o radioterapia, pacientes con dificultades visuales y/o cognitivas

* Durante todo el artículo se hace referencia a las pacientes en términos femeninos, ya que la incidencia del cáncer de mama en mujeres es mayor. Sin embargo, los hombres representan el $1 \%$ de la incidencia y en este trabajo también se cuenta con una participación cercana a dicho porcentaje. 
para responder a los cuestionarios y aquellas personas que por su estado de salud o condiciones sociales no puedan realizar una entrevista de aproximadamente una hora de duración.

Todas las pacientes firman el consentimiento informado previa inclusión en el estudio.

Desde junio de 2017 hasta marzo del 2020, se ha ofrecido participar a 274 pacientes que cumplían los criterios de inclusión, de las cuales sólo una rechazó participar en el estudio. De las 273 que firmaron el consentimiento informado 16 no llegaron a realizar la entrevista: 2 por causas de salud y 14 por motivos personales. Por lo que la muestra final a fecha de marzo 2020 es de 257 pacientes. Ver Figura 1

\section{Procedimiento}

Las personas entrevistadoras del equipo se encargan de administrar una serie de instrumentos y de realizar las entrevistas semiestructuradas a las pacientes de cáncer de mama del Servicio de Oncología Médica del Institut Català d'Oncologia (ICO) de l'Hospitalet de Llobregat, Barcelona, España. Ver Figura 2

Los criterios que se han utilizado para seleccionar a las personas entrevistadoras son los siguientes: a) estudiantes del Grado de Psicología de la Universitat Autònoma de Barcelona (UAB) que estén cursando el último año; o estudiantes del Máster Universitario de Psicología General y Sanitaria o del Máster de Investigación en Psicología Clínica y de la Salud; b) con interés y conocimientos previos en Psicooncología; c) proximidad geográfica para poder acudir a las reuniones presenciales realizadas por el equipo. Dichas entrevistadoras reciben una formación específica y conjunta en formato role-playing por parte del equipo principal, el cual está formado por: una oncóloga $(\mathrm{CF})$, dos psicooncólogos (AF y ER), dos estadísticas (ST y FM) y una epidemióloga (MM). El objetivo del entrenamiento previo es sistematizar la administración de los cuestionarios y la realización de las entrevistas. De este modo se pretende evitar sesgos por parte de los evaluadores. Además, para complementar el entrenamiento, las entrevistadoras reciben clases teóricas y prácticas durante varias semanas con la oncóloga en sus visitas programadas, con el fin de aproximarse a la situación médica de la población diana y familiarizarse con los términos médicos utilizados.

El protocolo del estudio fue aprobado por el comité de ética del Hospital Universitario de Bellvitge (HUB) en octubre de 2016. Los oncólogos médicos de la unidad se encargan de informar a las pacientes, que cumplen los criterios de inclusión previamente mencionados, sobre la investigación y las que están interesadas en participar firman el consentimiento informado. Las personas evaluadoras, con los datos proporcionados en el consentimiento informado, se encargan de llamar a las participantes para concertar las entrevistas presenciales. Antes de iniciar la entrevista, se vuelven a explicar los objetivos de la investigación, remarcando la confidencialidad y el anonimato de su colaboración. Las entrevistas son presenciales y tienen una duración de una hora aproximadamente y se estructuran de la siguiente manera: a) cuestionario de datos sociodemográficos y antecedentes psicosociales; $b$ ) se administran los cuestionarios de calidad de vida QLCA-AFex Font, QLQ-C30 y QLQ-BR23; c) se realiza la entrevista semiestructurada; d) se administran los cuestionarios de la escala de ansiedad y depresión hospitalaria (HADS), detección de malestar emocional (DME), la escala 
breve de afrontamiento resiliente (BRCS), la escala reducida de ajuste mental al cáncer (MINI-MAC), el test de optimismo disposicional versión revisada (LOT-R) y el test de optimismo estratégico $(\mathrm{OE})$. La información estrictamente clínica es recogida aparte por la oncóloga referente del estudio a través de las historias clínicas y se almacena de forma prospectiva en una base de datos separada de los datos recogidos por las personas evaluadoras. De esta manera se pretende mantener una valoración de la calidad de vida no sesgada y manteniendo el doble ciego. Dicha base de datos se integra en la base de datos de calidad de vida en el momento del análisis de forma codificada, de manera que sólo el oncólogo responsable de la paciente puede tener acceso a la identidad de la misma.

Figura 2. Diagrama descriptivo del diseño del estudio

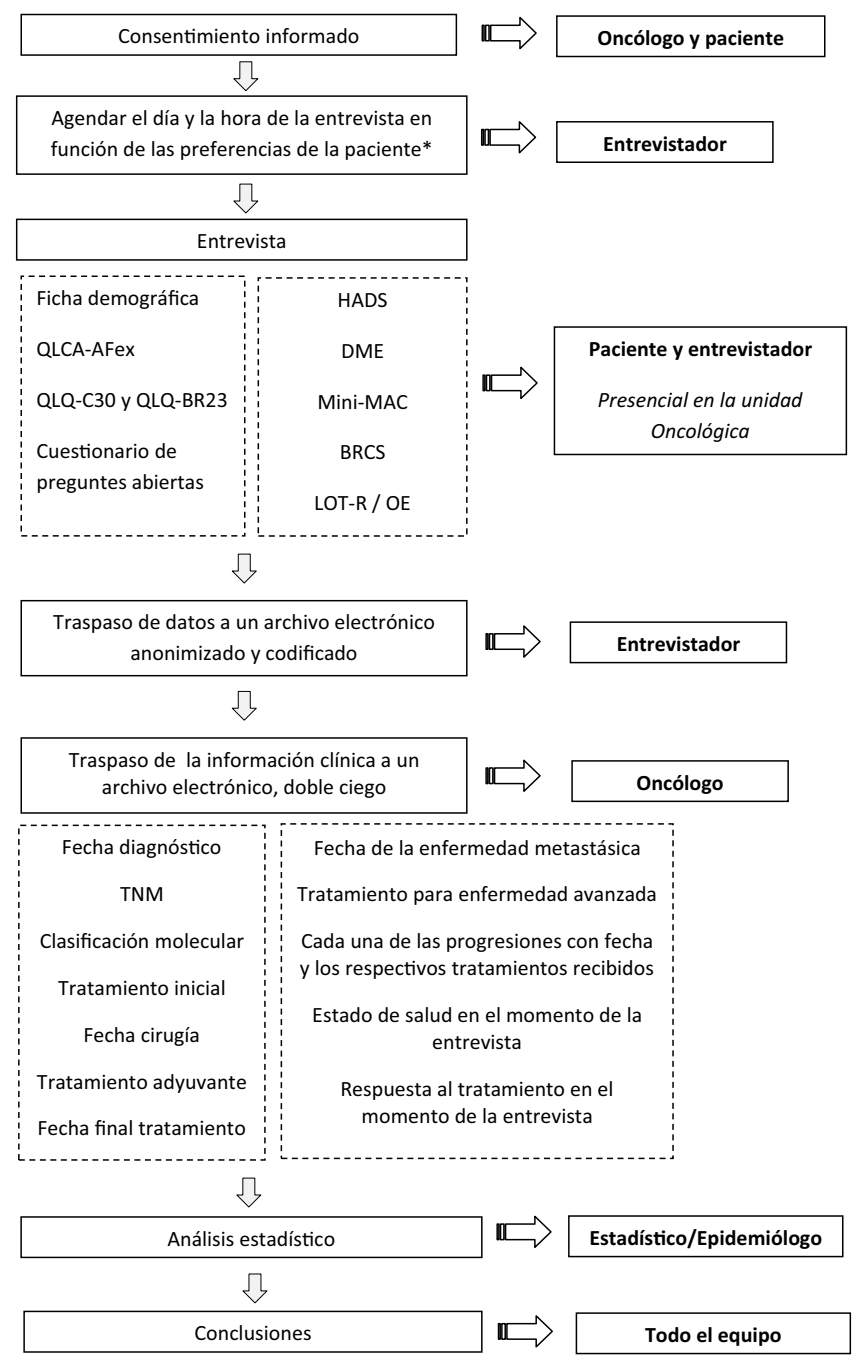

* A partir de este punto se repetirán los pasos en las futuras evaluaciones longitudinales 
En noviembre 2017 se hizo una revisión interna de la metodología y se aprobaron dos modificaciones del protocolo: se redujeron las preguntas de la entrevista semiestructurada que eran redundantes y se añadió el cuestionario de optimismo estratégico dado su interés y aplicabilidad clínica para complementar la información aportada por el optimismo disposicional. Los primeros resultados clínicos de la aplicación de dicho cuestionario fueron hechos públicos en septiembre 2017 y publicados posteriormente ${ }^{(15)}$.

\section{Instrumentos}

Cuestionario de datos sociodemográficos y antecedentes psicosociales

Se ha elaborado específicamente para esta investigación y recoge la edad, estado civil, nivel de estudios, situación laboral y genograma, preguntando específicamente si hay antecedentes familiares de cáncer de mama o de ovario. También se consulta sobre aspectos psicológicos, como los antecedentes personales de trastorno del estado de ánimo, si ha recibido en algún momento o está recibiendo actualmente algún tratamiento psicológico y/o psicofarmacológico, y si le gustaría recibir algún tipo de ayuda psico-social. Por último, se pregunta sobre los sucesos vitales estresantes que la paciente considera que ha vivido a lo largo de su vida.

Cuestionario de calidad de vida QLCA-AFex ${ }^{(16,17)}$

Este instrumento valora la calidad de vida de los pacientes oncológicos en régimen ambulatorio en relación con su estado de salud, centrándose en la última semana. Se trata de un instrumento elaborado y validado con población española y que para su validación incluyó a 404 pacientes en diferentes estadios de la enfermedad ${ }^{(16,18)}$. Está construido por 27 ítems que se puntúan de 0 a 100 en una escala de respuesta análogo-visual. Los ítems se agrupan en cuatro subescalas: síntomas y efectos secundarios (6 ítems), disminución de la autonomía personal (5 ítems), dificultades familiares y sociales ( 8 ítems), y malestar emocional/psicológico ( 7 ítems). Además de un último ítem que valora la calidad de vida global, cogiendo como referencia la última semana. Las dos primeras dimensiones valoran el impacto directo de la enfermedad y se agruparían como malestar corporal. En cambio, las otras dos son más indirectas, midiendo el malestar psicosocial. La puntuación media de cada subescala se obtiene calculando el sumatorio de las respuestas a los ítems que componen cada escala, dividida por el número de ítems de ésta. Así, una mayor puntuación en una determinada subescala significa mayor pérdida de calidad de vida relacionada con el aspecto valorado por ésta, y, por tanto, que esos elementos que la forman sufren más afectación. Finalmente, los resultados cuantitativos se pueden agrupar de manera cualitativa en 3 categorías: nada o muy poca afectación (valores de 5 o menos), bastante afectación (de 6 a 50) y mucha afectación (mayores a 50) ${ }^{(17)}$.

\section{Cuestionarios QLQ-C30(19) y QLQ-BR23(20)}

Ambos instrumentos han sido desarrollados por la European Organization for Research and Therapy of Cancer (EORTC). Dichos cuestionarios son muy utilizados para medir la calidad de vida en pacientes con cáncer de mama ${ }^{(21)}$. Además, han sido traducidos a muchos idiomas y se dispone de valores normativos para la gran mayoría de países europeos ${ }^{(22)}$, lo que nos permite poder realizar comparaciones internacionales. 
La versión 3 del QLQ-C30 consta de 30 ítems y evalúa cinco áreas funcionales (funcionamiento físico, rol, emocional, social y cognitivo); tres subescalas sintomáticas (astenia, dolor y náuseas y vómitos); ítems de síntomas (disnea, insomnio, pérdida del apetito, estreñimiento y diarrea), juntamente con el impacto económico del cáncer y del tratamiento y con una subescala de calidad de vida. A una mayor puntuación en las subescalas funcionales y en la global le corresponde una mejor calidad de vida y cuanto mayor sea la puntuación en escalas de síntomas y en los síntomas específicos peor será la calidad de vida ${ }^{(23)}$.

Para completar la información y que ésta fuera más específica y sensible para el cáncer de mama, también fue empleado el QLQ-BR23 de 23 ítems que valora áreas relacionadas con las diferentes modalidades de tratamiento oncológico, efectos secundarios y otros aspectos de la calidad de vida más específicamente afectados en el cáncer de mama, como la imagen corporal y la sexualidad. Las puntuaciones van de 0 a 100 y a mayor puntuación, mayor sintomatología ${ }^{(24)}$.

Escala de Ansiedad y Depresión Hospitalaria (HADS) ${ }^{(25)}$

La escala HADS es un instrumento auto-aplicado formado por 14 ítems. Consta de dos subescalas (HADA y HADD) de 7 ítems cada una, que puntúa en una escala Likert de 0 a 3 y con un rango de puntuaciones por subescala de 0 a 21. A mayor puntuación, más ansiedad y depresión. Las puntuaciones superiores a once para cada subescala indicarían "caso" y mayores de ocho se considerarían "caso probable"(26).

\section{Cuestionario de Detección del Malestar Emocional (DME) $)^{(27)}$}

El cuestionario para detectar el malestar emocional (DME) consta de dos partes. La primera contiene tres preguntas dirigidas a la persona enferma, dos de ellas en formato de escala visual numérica de 0 a 10 , en las que se evalúa el estado de ánimo y la percepción de afrontamiento de la situación, y otra que registra la presencia o ausencia de preocupaciones. La segunda parte consiste en una observación, por parte del profesional sanitario, de la presencia de signos externos de malestar emocional, en donde se registra su existencia. El DME otorga una puntuación total ( 0 a 20$)$ formada por la suma de las puntuaciones de las respuestas a las preguntas referentes a la valoración del estado de ánimo y a la de cómo lleva la situación. Las preguntas sobre preocupaciones o signos externos de malestar emocional permiten a los profesionales sanitarios llevar a cabo una atención más específica. Según los autores, las puntuaciones iguales o superiores a 9 puntos indican malestar emocional moderado, y superiores a 13, malestar severo. Se ha confirmado la validez clínica del instrumento como herramienta de cribado de malestar emocional en los enfermos oncológicos avanzados ${ }^{(28)}$.

Escala reducida de ajuste mental al cáncer (MINI-MAC) ${ }^{(29)}$

Instrumento de 29 ítems que evalúa las respuestas cognitivas y conductuales al cáncer en una escala Likert de 4 puntos y que diferencia 5 estilos de afrontamiento: espíritu de lucha, indefensión, preocupación ansiosa, fatalismo y evitación ${ }^{(29,30)}$. La versión extensa de este cuestionario forma parte de la propuesta de la Sociedad Internacional de Psico-oncología (IPOS) para medir la respuesta al cáncer. La razón para elegir Mini-MAC en lugar de la escala completa MAC es facilitar su administración y no alargar el tiempo de las entrevistas cuando ya se puede disponer de la información esencial. 
Escala Breve de Afrontamiento Resiliente; Brief Resilient Coping Scale (BRCS) ${ }^{(31)}$

El objetivo de este instrumento auto-administrado es evaluar la capacidad de las personas para manejar el estrés, provocado por la situación, de manera adaptativa. Se compone de 4 ítems diseñados para medir las tendencias de afrontamiento resiliente al estrés, con 5 categorías de respuesta tipo Likert en una escala de 1 al 5, donde (1) significa que la declaración "no me describe en absoluto", (2) "me describe un poco", (3) "ni mucho ni poco", (4) "me describe bastante", y (5) significa "me describe muy bien". El rango de puntuaciones resultantes del sumatorio es de 4 a 20, siendo una puntuación total, igual o inferior a 13 indicadora de baja resiliencia, e igual o superior a 17 de alta resiliencia, y de 14 a 16 media, según los autores ${ }^{(31)}$.

Test de optimismo disposicional. Life Orientation Test versión revisada (LOT-R) ${ }^{(32)}$

El test está formado por 10 ítems, 3 de los cuales están formulados en sentido positivo y expresan optimismo como tendencia, 3 están expuestos en sentido negativo (pesimismo) y 4 son de relleno. Los 3 ítems que miden el pesimismo deben invertirse en el momento de corregir la puntuación, dado que la puntuación conjunta se refiere al nivel de optimismo. Los ítems se puntúan en una escala Likert de 5 puntos, que va de 0 (totalmente en desacuerdo) a 4 (totalmente de acuerdo); obteniéndose así una puntuación total con rangos entre 0 y 30 puntos $^{(33)}$.

Test de optimismo estratégico $(\mathrm{OE})^{(15)}$

El constructo "optimismo estratégico" hace referencia al uso del optimismo como una estrategia cognitiva que permite adaptarse a la situación y mantener la calidad de vida según cómo se evalúe el presente, pasado y futuro ${ }^{(15)}$. Este cuestionario consta de 15 ítems agrupados en cuatro factores: Expectativas de resultado positivo y percepción de control ante la dificultad de salud; valoración positiva del pasado en el momento actual de salud; benefit finding a raíz de la enfermedad; y responsabilidad/ culpabilidad por la situación de salud. Los ítems están formulados como una escala Likert de 4 puntos, donde el 1 equivale a "ninguna vez" y el 4 a "continuamente", por lo que la puntuación total del cuestionario va de 15 a 60 puntos. Se invierte la corrección de dos ítems para que todas las puntuaciones sean positivas.

\section{Entrevista semiestructurada de preguntas abiertas}

La administración de los cuestionarios anteriormente comentados se completa con una entrevista semiestructurada de preguntas abiertas ya utilizada en un estudio previo $^{(13)}$ adaptada al estudio actual y que incluye aspectos médicos, psicosociales, emocionales, cognitivos y conductuales y las necesidades no atendidas (ver figura 3). De esta manera, se pretende recoger la experiencia y la opinión de las pacientes que no siempre queda reflejada en los cuestionarios cuantitativos. Las respuestas dadas a las diversas preguntas de la entrevista semiestructurada son transcritas literalmente por las personas evaluadoras con el fin de recoger la información de manera fidedigna. 
Figura 3. Entrevista semi-estructurada

\section{Aspectos médicos}

- Tratamiento

- ¿Qué espera del tratamiento?

- Efectos secundarios:

- ¿Qué efectos secundarios son los que más la limitan?

- ¿Qué hace para evitar los efectos secundarios en la medida de lo posible?

\section{Aspectos psicosociales}

- ¿Qué aspectos de la enfermedad le cuesta más afrontar?

- ¿Con quién comparte su malestar emocional?

- ¿Qué actividades disminuyen su malestar emocional?

- ¿Qué actividades diarias le ocupan más tiempo?

¿A qué querría dedicarle más tiempo?

¿Qué cosas querría eliminar de su carga diaria?

- ¿Hasta qué punto la enfermedad disminuye su calidad de vida?

- ¿Qué aspectos de la enfermedad empeoran su calidad de vida?

- ¿Qué aspectos de la enfermedad mejoran su calidad de vida?

- ¿De qué soporte socio-familiar dispone?

- Relaciones sociales (amigos/as, compañeros/as de trabajo,...).

- Relación con la familia (padres/madres, hijos/hijas, pareja,...).

- ¿Qué es lo que más le preocupa?

- ¿Considera que hay algún aspecto positivo o que ha aprendido alguna cosa de la enfermedad?

- ¿Cuáles son sus necesidades no atendidas?

- ¿Qué echa en falta en el sistema sanitario actual?

- ¿En qué la podríamos ayudar los profesionales de la salud?

\section{Análisis estadístico}

Para recopilar los datos codificados recogidos se ha utilizado el programa Microsoft Excel 2010 y para realizar la posterior explotación estadística de éstos se ha recurrido al programa STATA versión 14 para Windows (StataCorp. 2015. Stata Statistical Software: Release 14. College Station, TX: StataCorp LP) y al R Studio 3.5.1 software (R Foundation for Statistical Computing; Vienna, Austria). Se proporcionan los datos descriptivos de la serie con las distribuciones de frecuencias y los porcentajes de columnas para cada variable sociodemográficas y clínicas. Se ha analizado el cumplimiento del protocolo calculando las respuestas no contestadas o missings de cada cuestionario. Teniendo como referencia de calidad un porcentaje de missings inferior al $10 \%{ }^{(34)}$.

\section{Resultados}

Caracteristicas sociodemográficas

A continuación, se muestran las características sociodemográficas de la muestra. Ver Tabla 1 . 
El grupo está formado por 257 pacientes, la edad media es de 57,9 años con una desviación estándar de 10,1 y con edades comprendidas entre 25 y 82 años. La mayor parte de las pacientes tiene hijos $(87,9 \%)$ y está casada $(65,4 \%)$. Por lo que respecta a los estudios, un tercio de las participantes tiene estudios secundarios (37\%), seguidos de otro tercio que tiene estudios primarios $(36,6 \%)$ y finalmente el $25,3 \%$ tiene estudios superiores.

Tabla 1. Características descriptivas sociodemográficas de la muestra

\begin{tabular}{|c|c|c|}
\hline & \multicolumn{2}{|c|}{$\begin{array}{l}\text { Conjunto de la muestra } \\
\qquad(\mathrm{N}=\mathbf{2 5 7})\end{array}$} \\
\hline & $\mathrm{N}$ & $\%$ \\
\hline \multicolumn{3}{|l|}{ Edad (en años) } \\
\hline $\begin{array}{r}<40 \\
40-50 \\
50-65 \\
>65\end{array}$ & $\begin{array}{c}7 \\
52 \\
129 \\
69\end{array}$ & $\begin{array}{c}2,72 \\
20,23 \\
50,19 \\
26,85\end{array}$ \\
\hline \multicolumn{3}{|l|}{ Sexo } \\
\hline $\begin{array}{r}\text { Mujeres } \\
\text { Hombres }\end{array}$ & $\begin{array}{c}254 \\
3\end{array}$ & $\begin{array}{c}98,83 \\
1,17\end{array}$ \\
\hline \multicolumn{3}{|l|}{ Estado civil } \\
\hline $\begin{array}{r}\text { Casada } \\
\text { Divorciada } \\
\text { Pareja estable } \\
\text { Separada } \\
\text { Soltera } \\
\text { Viuda }\end{array}$ & $\begin{array}{c}168 \\
31 \\
16 \\
3 \\
18 \\
21\end{array}$ & $\begin{array}{c}65,37 \\
12,06 \\
6,23 \\
1,17 \\
7,00 \\
8,17\end{array}$ \\
\hline \multicolumn{3}{|l|}{ Estudios } \\
\hline $\begin{array}{r}\text { Primarios } \\
\text { Medios } \\
\text { Superiores } \\
\mathrm{NC}\end{array}$ & $\begin{array}{c}94 \\
95 \\
65 \\
3\end{array}$ & $\begin{array}{c}36,58 \\
36,96 \\
25,29 \\
1,17\end{array}$ \\
\hline \multicolumn{3}{|l|}{ Descendencia } \\
\hline $\begin{array}{r}\text { Sí } \\
\text { No }\end{array}$ & $\begin{array}{c}226 \\
31\end{array}$ & $\begin{array}{l}87,94 \\
12,06\end{array}$ \\
\hline Total & 257 & 100,00 \\
\hline
\end{tabular}

Abreviaciones: N: número de participantes; \%: porcentaje por columnas; NC: no contesta 


\section{Características clínicas}

La muestra está compuesta por pacientes en periodo libre de enfermedad que representan el 75,5\% $(\mathrm{n}=194)$ y pacientes con enfermedad avanzada o metastásica las cuales constituyen el 24,5\% $(n=63)$ restante. La muestra incluye pacientes en todos los estadios de la enfermedad y de todos los subtipos moleculares, siendo representativa de la población atendida en su conjunto en el Servicio de Oncología Médica. Ver Tabla 2.

Tabla 2. Características clínicas de la muestra

\begin{tabular}{|c|c|c|}
\hline & \multicolumn{2}{|c|}{$\begin{array}{l}\text { Conjunto de la muestra } \\
\qquad(\mathrm{N}=\mathbf{2 5 7})\end{array}$} \\
\hline & $\mathrm{N}$ & $\%$ \\
\hline \multicolumn{3}{|l|}{ Situación clínica } \\
\hline Libre de enfermedad & 194 & 75,49 \\
\hline Metástasis & 63 & 24,51 \\
\hline \multicolumn{3}{|l|}{ Estadio } \\
\hline 0 & 4 & 1,56 \\
\hline I & 67 & 26,07 \\
\hline IIA & 70 & 27,24 \\
\hline IIB & 50 & 19,46 \\
\hline IIIA & 30 & 11,67 \\
\hline IIIB & 10 & 3,89 \\
\hline IIIC & 11 & 4,28 \\
\hline IV & 15 & 5,84 \\
\hline \multicolumn{3}{|l|}{ Subtipo molecular } \\
\hline Luminal A & 79 & 30,74 \\
\hline Luminal B & 110 & 42,80 \\
\hline Luminal B HER2 & 26 & 10,12 \\
\hline HER2 & 17 & 6,61 \\
\hline Triple Negativo & 21 & 8,17 \\
\hline In situ & 4 & 1,56 \\
\hline \multicolumn{3}{|l|}{ Tratamiento en el momento de la entrevista } \\
\hline No tratamiento & 62 & 24,12 \\
\hline Quimioterapia & 11 & 4,28 \\
\hline Quimioterapia y anti-HER-2 & 10 & 3,89 \\
\hline Anti-HER-2 & 3 & 1,17 \\
\hline Hormonoterapia & 149 & 57,98 \\
\hline Hormonoterapia e inhibidores de ciclinas & 22 & 8,56 \\
\hline Total & 257 & $\mathbf{1 0 0 , 0 0}$ \\
\hline
\end{tabular}

Abreviaciones: N: Número de participantes; \%: porcentaje por columnas; HER-2: Human Epidermal Growth Factor Receptor

Respecto al tratamiento recibido en el momento de la entrevista, mayoritariamente es el tratamiento hormonal habiendo diferencias relevantes entre las pacientes metastásicas y no metastásicas debido a la distinta situación de salud de cada grupo. En el grupo de metastásicas estaban en respuesta al tratamiento en el momento de la entrevista el 76,2\% de las pacientes. Ver Tabla 3. 
Tabla 3. Tratamientos recibidos y respuesta en el momento de la entrevista por subgrupos

\begin{tabular}{|c|c|c|}
\hline & \multicolumn{2}{|c|}{$\begin{array}{l}\text { Conjunto de la muestra } \\
\qquad(\mathrm{N}=\mathbf{2 5 7})\end{array}$} \\
\hline & $\begin{array}{c}\text { No metastásicas } \\
\mathrm{N}=194 \\
\mathrm{~N}(\%)\end{array}$ & $\begin{array}{c}\text { Metastásicas } \\
\qquad \begin{array}{c}\mathrm{N}=63 \\
\mathrm{~N}(\%)\end{array}\end{array}$ \\
\hline \multicolumn{3}{|l|}{ Tratamiento en el momento de la entrevista } \\
\hline $\begin{array}{r}\text { No tratamiento } \\
\text { Quimioterapia } \\
\text { Quimioterapia y anti-HER-2 } \\
\text { Anti-HER-2 } \\
\text { Hormonoterapia } \\
\text { Hormonoterapia e inhibidores de ciclinas }\end{array}$ & $\begin{array}{c}61(31,4 \%) \\
0 \\
0 \\
0 \\
133(68,5 \%) \\
0\end{array}$ & $\begin{array}{c}1(1,6 \%) \\
11(17,5 \%) \\
10(15,9 \%) \\
3(4,8 \%) \\
16(25,4 \%) \\
22(34,9 \%)\end{array}$ \\
\hline \multicolumn{3}{|c|}{ Respuesta al tratamiento en el momento de la entrevista } \\
\hline $\begin{array}{r}\text { No aplica } \\
\text { Respuesta } \\
\text { No respuesta }\end{array}$ & $\begin{array}{c}194(100 \%) \\
0 \\
0\end{array}$ & $\begin{array}{c}0 \\
48(76,2 \%) \\
15(23,8 \%)\end{array}$ \\
\hline Total & $194(100 \%)$ & $63(100 \%)$ \\
\hline
\end{tabular}

Abreviaciones: N: Número de participantes; \%: porcentaje por columnas; HER-2: Human Epidermal Growth Factor Receptor

\section{Desarrollo de la entrevista y aceptación por parte de las pacientes}

Las entrevistadoras conducen cada entrevista sin condicionar las respuestas, el hecho de que sea presencial crea un ambiente distendido y de confianza donde la paciente pueda revivir su experiencia. El objetivo de esta entrevista es recoger de la forma más fidedigna la calidad de vida de las pacientes y sus estrategias de afrontamiento. En ningún momento es una maniobra terapéutica. Con todo, la experiencia llevando a cabo este estudio es que las pacientes agradecen que se les brinde la oportunidad de hacer balance de esta experiencia vital tan impactante. Es cierto que la entrevista es extensa, debido a que se han incluido muchos instrumentos de calidad de vida y de medidas de afrontamiento, pero las pacientes entienden que es una necesidad del estudio y a su vez agradecen el tiempo dedicado a su historia personal.

Finalmente, las participantes expresan asimismo su satisfacción por participar en un ensayo clínico que pueda ayudar a otras pacientes que tengan que pasar por dicha experiencia. La entrevista de preguntas semiestructurada además les permite expresar preocupaciones ajenas a la enfermedad oncológica, que no quedan reflejadas en los instrumentos de calidad de vida convencionales y que enriquecen la valoración holística de la calidad de vida de las pacientes y permite entender mejor su situación real. 


\section{Valor añadido de la entrevista presencial}

Las entrevistadoras han observado un denominador común en la mayoría de las pacientes entrevistadas: esfuerzo de continuar adelante, la capacidad de vivir con la incertidumbre y el cambio en la perspectiva vital. Cada una de las participantes tiene una historia vital y de la enfermedad particular, personal e íntima, y unas estrategias y habilidades individuales, por eso, es tan importante esforzarse en escucharlas una a una, para poder elaborar y brindar una ayuda que se adecue a sus necesidades. Este protocolo presencial da unas guías de cómo proceder para que este hecho sea realidad, permitiendo recoger muchos datos objetivos cuantificables, pero también el valor subjetivo de toda la vivencia de la enfermedad a lo largo de todo su curso evolutivo, con todos sus matices y ámbitos de la vida implicados.

\section{Descripción de las respuestas no contestadas o missings}

En ninguno de los cuestionarios se ha obtenido un porcentaje superior al $10 \%$ de respuestas no contestadas o missings ${ }^{(34)}$. Concretamente, la escala con más missings ha sido la escala DME: donde 20 personas han dejado alguna respuesta en blanco, lo que representa el 7,8\% del total de pacientes incluidas hasta ahora en el estudio. En comparación, en los cuestionarios BRCS y LOT-R se observan menos omisiones, tan solo 2 pacientes tienen missings en estos tests, siendo el $0,8 \%$ del total de la muestra. Cabe remarcar que, siendo cuestionarios pensados para mujeres, los hombres incluidos en el estudio han contestado a todas las preguntas sin missings.

\section{Discusión}

El cáncer de mama es considerado una enfermedad crónica que impacta de modo importante en la calidad de vida de las pacientes. Actualmente, no existe un procedimiento estándar que la evalúe teniendo en cuenta las diferentes situaciones médicas por las que pueden pasar las pacientes. El presente estudio ha tenido como objetivo describir la metodología utilizada por el equipo de investigación, a fin de elaborar un procedimiento evaluativo de la calidad de vida, factible de ser administrado de manera prospectiva, que incluya variables moduladoras de la calidad de vida, tanto las descritas en la literatura como las obtenidas a partir de las opiniones de nuestras propias pacientes, incluyendo tanto pacientes en período libre de enfermedad como metastásicas.

Actualmente, no existe un acuerdo unánime en cuanto a la definición y conceptualización de la calidad de vida ${ }^{(35)}$. Dicha falta de claridad en la definición del concepto implica una falta de consenso en las estrategias empleadas para su medición ${ }^{(35,36)}$. En el presente estudio se parte de la definición de la Organización Mundial de la Salud (OMS) para la calidad de vida, la cual la conceptualiza como un constructo multidimensional en el que se tienen en cuenta las percepciones que muestra un individuo sobre su lugar en la existencia, en el contexto de la cultura y del sistema de valores en los que vive, en relación con sus objetivos, expectativas, normas y preocupaciones ${ }^{(37)}$.

Al tratarse de un constructo multidimensional, existen una serie de variables psicológicas que se asocian a una buena calidad de vida en pacientes con cáncer de 
mama; entre ellas se encuentra la resiliencia ${ }^{(38)}$, las estrategias de afrontamiento $^{(39)}$ y el optimismo, tanto el disposicional ${ }^{(40)}$ como el estratégico ${ }^{(15,41)}$. Por ello, la identificación de estas variables puede ayudar a modular la calidad de vida de estas pacientes y detectar los factores protectores que la preservan.

En este momento, se dispone de múltiples instrumentos para medir la calidad de vida en pacientes oncológicos; entre ellos cabe destacar el de la EORTC QLQBR2323 y el FACT-B24. Sin embargo, no hay indicaciones de si estos instrumentos son adecuados para todas las etapas de la enfermedad ${ }^{(42)}$. Este es un aspecto a tener en cuenta en pacientes con enfermedad avanzada, ya que a menudo, dichos instrumentos están más orientados a la enfermedad inicial, haciendo más énfasis en los aspectos físicos de la cirugía y los efectos agudos de los tratamientos y recogen en menor medida las preocupaciones que impactan en la calidad de vida de las pacientes con enfermedad avanzada, como por ejemplo, el final de vida o el cuidado de los hijos cuando ellas falten. Hay que tener en cuenta que las enfermas metastásicas tienen necesidades diferentes en comparación con las diagnosticadas en una etapa temprana y que el impacto emocional del diagnóstico de enfermedad metastásica, con el consiguiente significado de incurabilidad inherente, difícilmente puede ser recogido utilizando únicamente escalas de calidad de vida desarrolladas fundamentalmente para otras etapas clínicas de la enfermedad ${ }^{(13,42)}$.

Por otra parte, es necesario tener en cuenta el entorno sociocultural en que se desarrollan los sistemas de evaluación. La tendencia imperante de adaptar instrumentos desde el entorno anglosajón a nuestro entorno puede conllevar, pese a los esfuerzos de las técnicas psicométricas, a que se pierdan o no se consideren suficientemente aspectos vinculados a la cultura. Además, los cuestionarios actuales de calidad de vida tienen varias limitaciones como describe Irarrázaval et al. (2013), quien apunta que generalmente son auto-administrados y en muchas ocasiones hay menor accesibilidad a las evaluaciones por parte de los pacientes más graves. Por otra parte, acostumbran a aplicarse en un momento muy concreto de la enfermedad de forma puntual, con lo que se pierde la capacidad de observar la evolución de la calidad de vida de las pacientes a lo largo del tiempo ${ }^{(43)}$.

Por consiguiente, el estudio descrito tiene el objetivo a largo plazo de desarrollar un sistema de evaluación continuado de la calidad de vida, que represente a todas las pacientes con cáncer de mama independientemente de su estadio de enfermedad y que recoja la máxima información con el menor desgaste posible para ellas. De este modo, se pretende disponer de un sistema de valoración factible, para conocer de manera sistemática la calidad de vida de las pacientes en los diferentes estadios de la enfermedad teniendo en cuenta la percepción subjetiva de cada persona y que se pueda comparar y aplicar de forma periódica para ver la evolución de la percepción de la calidad de vida a lo largo del tiempo. Por otra parte, se pretende poder detectar el proceso de adaptación de las pacientes oncológicas, incluyendo algunas medidas de resiliencia, optimismo y afrontamiento. Es decir, implementar una valoración prospectiva que permita observar y fomentar diferentes aspectos asociados a la adaptación y a la calidad de vida a largo plazo. Además, puede ser un sistema de valoración que también permite explorar y reflejar las necesidades no atendidas de las pacientes y sus preocupaciones, complementando la información recogida en los cuestionarios de calidad de vida existentes.

Al plantearse como un estudio longitudinal, presenta la potencialidad de poder medir la evolución de la calidad de vida de las pacientes con una misma batería de 
instrumentos a lo largo de la enfermedad, para conocer, qué aspectos de la calidad de vida mejoran, cuales se mantienen, y cuales pueden empeorar, no sólo por el paso del tiempo, sino también ante los cambios en el estado de salud de las mismas pacientes. Por ejemplo, en las entrevistas algunas pacientes refieren una falta de comprensión por parte de los profesionales de la salud y de su entorno familiar acerca de las secuelas que persisten a lo largo del tiempo, más allá de la caída del cabello o la debilidad del brazo después de la linfadenectomía como la fatiga, la falta de concentración, el insomnio, la pérdida de la libido o la pérdida de ilusión por las cosas. Estas secuelas no son tan conocidas ni difundidas, pero son aspectos con los que las pacientes conviven en muchas ocasiones por el resto de sus vidas ${ }^{(44)}$.

Estamos en un contexto donde el contacto es importante y una de las cosas que hemos podido aprender con este estudio, es que las pacientes agradecen que les proporcionemos un espacio donde se sientan escuchadas y comprendidas. Es por ello que, aunque el objetivo sea crear un sistema de valoración reducido en el tiempo, recomendamos que sea presencial.

Finalmente, recalcar que este trabajo brinda la oportunidad de explicar y conocer el día a día de las pacientes, su estado actual y el proceso de enfermedad que han vivido, al mismo tiempo que se da visibilidad a la enfermedad en etapa avanzada. Siendo uno de los deberes de los profesionales que trabajan en este campo, plantear estudios e instrumentos para ayudar a comprender mejor y con mayor precisión a las personas tratadas, investigando y creando nuevo conocimiento, para así poder llegar a una práctica clínica más empática e integradora, contemplando muchas veces aspectos olvidados o incomprendidos.

No obstante, el presente estudio muestra también ciertas limitaciones que cabe señalar. Una de las más importantes es el problema de la participación a largo plazo, especialmente de la población metastásica, por la mortalidad asociada a dicho diagnóstico de incurabilidad. Se puede perder muestra con el paso del tiempo, hecho que puede afectar a la representatividad inicial ${ }^{(45)}$.

\section{Conclusión y perspectivas de futuro}

El protocolo multidimensional expuesto en este trabajo permitirá hacer un diagnóstico integral y diferencial de la calidad de vida de las pacientes con cáncer de mama, así como reflejar las necesidades no atendidas y las preocupaciones que muestran estas pacientes tanto en período libre de enfermedad como en estadio avanzado, complementando los sistemas de valoración actualmente disponibles.

Es importante conocer la calidad de vida de las pacientes con cáncer de mama y el grado de malestar emocional que no siempre es proporcional a la gravedad clínica, sino con la percepción subjetiva de gravedad que tiene cada paciente de sí misma. Por ello, a partir de la experiencia clínica vivencial y teniendo en cuenta las limitaciones de los instrumentos individuales, planteamos como orientación futura el desarrollo de un sistema conciso e integrado de diferentes instrumentos con los ítems más útiles y procurando que abarquen la complejidad de la enfermedad en sus diferentes fases. A partir del protocolo que continúa en fase de elaboración ahora prospectiva, esperamos poder contribuir a conocer mejor cómo viven nuestras pacientes con cáncer de mama, más allá de los aspectos físicos que comporta la enfermedad. La difusión de esta realidad puede ser útil no sólo para los profesionales 
de la esfera sanitaria que tratan con dichas pacientes, como oncólogos, ginecólogos, médicos de atención primaria, radioterapeutas, o psicólogos, sino también para que la población general conozca y pueda entender mejor la complejidad vivencial del cáncer de mama.

\section{Agradecimientos}

Agradecer la participación de cada una de las pacientes que han compartido su vivencia y experiencia en el estudio y que después de tres años desde su inicio lo continúan haciendo. Especialmente a aquellas pacientes que ya nos han dejado, pero no sin habernos dado una lección de vida con su actitud hacia la enfermedad y la vida, personas anónimas para la sociedad, pero igualmente heroínas de nuestro tiempo. Del mismo modo, agradecer a Pau Edo, Sara Arévalo, Cristina Fernández y Vicky Pérez su plena dedicación en este proyecto durante el tiempo que estuvieron colaborando en la recogida de datos.

\section{Financiación}

Esta investigación no recibió ninguna subvención específica de agencias de financiamiento de sectores públicos ni privados o sin ánimo de lucro. Fue posible por dedicación propia gracias al Servicio de Oncología Médica del Institut Català d'Oncologia (ICO) y la Facultad de Psicología de la Universitat Autònoma de Barcelona (UAB).

\section{Referencias bibliograficas}

1. Talepasand S, Mahfar F. Relationship between defense mechanisms and the quality of life in women with breast cancer. Int J Cancer Manag 2018;11:1-8. doi: 10.5812/ijcm.11116

2. Bray F, Ferlay J, Soerjomataram I, Siegel RL, Torre LA, Jemal A. Global cancer statistics 2018: GLOBOCAN estimates of incidence and mortality worldwide for 36 cancers in 185 countries. CA Cancer J Clin 2018;68:394-424. doi: 10.3322/caac.21492

3. Asociación Española Contra el Cáncer. Pronóstico del Cáncer de Mama: Mortalidad y Esperanza de vida [Internet]. 2018 [citado 20 de noviembre de 2019]. Disponible en: https://www.aecc.es/es/todo-sobre-cancer/tipos-cancer/cancer-mama/mas-informacion/ evolucion-cancer-mama

4. Izquierdo A, Gispert R, Saladie F, Espinàs JA. Análisis de la incidencia, la supervivencia y la mortalidad según las principales localizaciones tumorales, 1985-2019: cáncer de mama. Med Clínica 2008;131:50-2. doi: 10.1016/S0025-7753(08)76433-9

5. Koch L, Jansen L, Herrmann A, Stegmaier C, Holleczek B, Singer S, et al. Quality of life in long-term breast cancer survivors - a 10-year longitudinal population-based study. Acta Oncol 2013;52:1119-28. doi: 10.3109/0284186X.2013.774461

6. Cardoso F, Costa A, Senkus E, Aapro M, André F, Barrios CH, et al. 3rd ESO-ESMO International Consensus Guidelines for Advanced Breast Cancer (ABC 3). Ann Oncol 2017;28:16-33. doi:10.1093/annonc/mdw544 
7. Cardoso F. Metastatic breast cancer patients: The forgotten heroes!. Breast 2009;18:2712. doi: 10.1016/j.breast.2009.09.001

8. Aziz NM. Cancer survivorship research: State of knowledge, challenges and opportunities. Acta Onco. 2007;46:417-32. doi: 10.1080/02841860701367878

9. Costa-Requena G, Rodríguez A, Fernández-Ortega P. Longitudinal assessment of distress and quality of life in the early stages of breast cancer treatment: Breast cancer and longitudinal distress. Scand J Caring Sci 2013;27:77-83. doi: 10.1111/j.14716712.2012.01003.x

10. Hernández F, Landero R. Aspectos psicosociales relacionados con la calidad de vida en mujeres con cáncer de mama Psychosocial aspects related to quality of life in women with breast cancer. Summa Psicológica 2014;11:99-104.

11. Hagedoorn M, Sanderman R, Bolks HN, Tuinstra J, Coyne JC. Distress in couples coping with cancer: A meta-analysis and critical review of role and gender effects. Psychol Bull 2008;134:1-30. doi: 10.1037/0033-2909.134.1.1

12. Arraras JI, Illarramendi JJ, Manterola A, Domínguez Á. Progresos en la evaluación de la calidad de vida en el cáncer de mama. El sistema de medida de la EORTC. Psicooncología 2007;4:367-84.

13. Falo C, Villar H, Rodríguez E, Mena M, Font A. Calidad de vida en cáncer de mama metastásico de larga evolución: La opinión de las pacientes. Psicooncología 2014;11:31331. doi: 10.5209/rev_PSIC.2014.v11.n2-3.47391

14. Ferlay J, Shin H-R, Bray F, Forman D, Mathers C, Parkin DM. Estimates of worldwide burden of cancer in 2008: GLOBOCAN 2008. Int J Cancer 2010;127:2893-917. doi: 10.1002/ijc. 25516

15. Pastells S, Font A. Propuesta de un instrumento para la detección del optimismo estratégico en cáncer de mama. Psicooncología 2019;16:315-28. doi: 10.5209/rev_PSIC.2014.v11. $\mathrm{n} 1.44914$

16. Font A. Valoración de la calidad de vida en pacientes de cáncer. [Tesi Doctoral]. [Bellaterra, Barcelona]: Universitat Autònoma de Barcelona.; 1988.

17. Font A. Cáncer y calidad de vida. Anu Psicol 1994;61:41-50.

18. Padierna C, Fernández C, González A. Calidad de vida en pacientes oncológicos terminales, medida con el QL-CA-AFEX. Psicothema 2002;14:1-8.

19. Aaronson NK, Ahmedzai S, Bergman B, Bullinger M, Cull A, Duez NJ, et al. The European Organization for Research and Treatment of Cancer QLQ-C30: A quality-oflife instrument for use in international clinical trials in oncology. JNCI J Natl Cancer Inst 1993;85:365-76.

20. Sprangers MA, Groenvold M, Arraras JI, Franklin J, te Velde A, Muller M, et al. The European Organization for Research and Treatment of Cancer breast cancer-specific quality-of-life questionnaire module: first results from a three-country field study. J Clin Oncol 1996;14:2756-68. doi: 10.1200/JCO.1996.14.10.2756

21. Finck C, Barradas S, Zenger M, Hinz A. Quality of life in breast cancer patients: Associations with optimism and social support. Int J Clin Health Psychol 2018;18:27-34. doi: 10.1016/j.ijchp.2017.11.002

22. Hinz A, Singer S, Brähler E. European reference values for the quality of life questionnaire EORTC QLQ-C30: Results of a German investigation and a summarizing analysis of six European general population normative studies. Acta Oncol. 2014;53:958-65. doi: 10.3109/0284186X.2013.879998

23. Cerezo O, Oñate-Ocaña LF, Arrieta-Joffe P, González-Lara F, García-Pasquel MJ, Bargalló-Rocha E, et al. Validation of the Mexican Spanish version of the EORTC QLQ 
C30 and BR23 questionnaires to assess health related quality of life in Mexican women with breast cancer. Eur J Cancer Care (Engl). 2012;21:684-691. doi: 10.1111/j.13652354.2012.01336.x

24. Arraras JI, Tejedor M, Illaramendi J j., Vera R, Pruja E, Marcos M, et al. The EORTC breast cancer quality questionnaire (QLQ-BR23): A psychometric study with Spanish patients. Psicol Conduct 2001;9:81-97.

25. Zigmond AS, Snaith RP. The hospital anxiety and depression scale. Acta Psychiatr Scand. 1983;67:361-70. doi: 10.1111/j.1600-0447.1983.tb09716.x.

26. Terol M, López-Roig S, Rodríguez-Marín J, Gelabert M, Pastor M-Á, Reig MT. Propiedades psicométricas de la Escala Hospitalaria de Ansiedad y Estrés (HAD) en población española. Ansiedad Estrés 2007;13:163-76.

27. Maté J, Mateo D, Bayés R, Bernaus M, Casas C, González-Barboteo J, et al. Elaboración y propuesta de un instrumento para la detección de malestar emocional en enfermos al final de la vida. Psicooncología 2009;6:507-18.

28. Limonero JT, Mateo D, Maté-Méndez J, González-Barboteo J, Bayés R, Bernaus M, et al. Evaluación de las propiedades psicométricas del cuestionario de Detección de Malestar Emocional (DME) en pacientes oncológicos. Gac Sanit 2012;26:145-52. doi: 10.1016/j. gaceta.2011.07.016

29. Watson M, Law M, dos Santos M, Greer S, Baruch J, Bliss J. The Mini-MAC: Further development of the Mental Adjustment to Cancer Scale. J Psychosoc Oncol 1994;12:3346. doi: 10.1300/J077V12N03_03

30. Calderón C, Carmona-Bayonas A, Hernández R, Ghanem I, Castelo B, Martinez de Castro $\mathrm{E}$, et al. Effects of pessimism, depression, fatigue, and pain on functional health-related quality of life in patients with resected non-advanced breast cancer. Breast 2019;44:10812. doi: 10.1016/j.breast.2019.01.012

31. Sinclair VG, Wallston KA. The development and psychometric evaluation of the Brief Resilient Coping Scale. Assessment 2004;11:94-101. doi: 10.1177/1073191103258144.

32. Otero JM, Luengo A, Romero E, Gómez JA, Castro C. Psicología de personalidad. Manual de prácticas. Barcelona: Ariel Practicum; 1998.

33. Ferrando PJ, Chico E, Tous JM. Propiedades psicométricas del test de optimismo Life Orientation Test. Psicothema. 2002;14:673-80.

34. Molenberghs G, Kenward M. Missing data in clinical studies. Chichester, UK: John Wiley and Sons Ltd; 2007.

35. Moons P, Budts W, De Geest S. Critique on the conceptualisation of quality of life: A review and evaluation of different conceptual approaches. Int J Nurs Stud 2006;43:891901. doi: 10.1016/j.ijnurstu.2006.03.015

36. Sánchez-Pedraza R, Ballesteros MP, Anzola JD. Concepto de calidad de vida en mujeres con cáncer. Rev Salud Pública 2010;12:889-902.

37. WHO Quality of Life Assessment Group. ¿Qué calidad de vida? / Grupo de la OMS sobre la calidad de vida. Foro Mund Salud 1996;174):385-7.

38. Rowland JH, Baker F. Introduction: Resilience of cancer survivors across the lifespan. Cancer. 2005;104(S11):2543-8. doi: 10.1002/cncr.21487

39. Derogatis LR, Abeloff MD, Melisaratos N. Psychological coping mechanisms and survival time in metastatic breast cancer. JAMA 1979;242:1504-8. doi: 10.1001/ jama.1979.03300140020016

40. Scheier MF, Carver CS. Optimism, coping, and health: assessment and implications of generalized outcome expectancies. Health Psychol 1985;4:219-47. doi: 10.1037//02786133.4.3.219 
41. Seligman MEP. Aprenda Optimismo: Haga de la vida una experiencia maravillosa. Barcelona: Clave, 2011.

42. Reed E, Kössler I, Hawthorn J. Quality of life assessments in advanced breast cancer: should there be more consistency?: Quality of life assessment in advanced breast cancer. Eur J Cancer Care (Engl) 2012;21:565-80. doi: 10.1111/j.1365-2354.2012.01370.x

43. Irarrázaval ME, Rodríguez PF, Fasce G, Silva FW, Waintrub H, Torres C, et al. Calidad de vida en cáncer de mama: validación del cuestionario BR23 en Chile. Rev Médica Chile 2013;141:723-34.

44. Rosedale M, Fu MR. Confronting the unexpected: temporal, situational, and attributive dimensions of distressing symptom experience for breast cancer survivors. Oncol Nurs Forum 2010;37:E28-33.

45. Caruana EJ, Roman M, Hernández-Sánchez J, Solli P. Longitudinal studies. J Thorac Dis 2015;7:E537-40. doi: 10.3978/j.issn.2072-1439.2015.10.63 